\title{
Non-thernial electrons and stellar radio emission
}

\author{
S. M. Whise and M. R. Kundu \\ Astronumy Program, Univ. of Mlaryland, College Patk, MD 20742
}

\begin{abstract}
ABSIRACT
Radio emission from dMe fline stars has both a flaring and a quiescent component. When we compare stellar radio emission with the Sull, however, we find that the apparent brightness temperature of the quiescent component often exceeds the temperature of non-themal solar radio flares, and so it is likely that stellar quiescent emission also comes from non-thermal electrons. The duration of stcllar quiescent cmission is much longer than solar monthernal emission. Obvious questions to ask are, what is the source of the non-thermal electrons, where do llicy reside, and how can non-lhemal emission last so long? Here we briclly review the observations of quiescent emission, argue that the emilting regions are small, show that such small regions can still account for the observed fluxes, and discuss the source of electrons.
\end{abstract}

\section{Quiescent emission from UV Celi and EQ Peg}

The first observation of llare stars at the VLA detected quiescent emission from UV Ceti at $6 \mathrm{~cm}$, corresponding to a brightness temperature of about $10^{8}\left(\mathrm{R} / \mathrm{R}_{\text {. }}\right)^{2} \mathrm{~K}$ (Gary and linsky. 1981). The flux from this star at $6 \mathrm{~cm}$ hals never been seen below $0.9 \mathrm{mJy}$, and the quicsecm component varies up to $3 \mathrm{mJy}$. The quiescent llux is unpolarized and slowly varying (i.c., variable on a limescalc of an hour or so). Quiescent flux may also be detected at $20 \mathrm{~cm}$, but seems to be much more variable there than at $6 \mathrm{~cm}$. In particular, while the ratio of $6 \mathrm{~cm}$ flux 1020 $\mathrm{cm}$ llux is usually in excess of unity, indicating optically thick emission, there have been a number of occasions on which the apparent $20 \mathrm{~cm}$ quiescent llux exceeds the $6 \mathrm{~cm}$ quiescent flux. Since flaring is more common at $20 \mathrm{~cm}$ than at $6 \mathrm{~cm}$, it is often difficult 10 decide what part of the observed $20 \mathrm{~cm}$ flux is actually quiescent.

Normally EQ Peg A is a reliable quiescent source at a level of about $0.5 \mathrm{mJy}$ at $6 \mathrm{~cm}$, corresponding to a brighuness temperature of $310^{8}\left(R_{R} / R_{*}\right)^{-2} \mathrm{~K}$. However it has shown onc large outburst which was clearly quiescent emission at a level 20 times nomal. This was during simultancous $X$-ray and radio observations by Kundu et al. (1988) on 1985 August 6 . At the beginning of the observation EXOSA'T detected the largest $X$-ray flare seen from this class of sliar. The VLA began observing half an hour later, and found the $6 \mathrm{~cm}$ flux from l:() Peg $A$ to be at about $10 \mathrm{mJy}$ at $6 \mathrm{~cm}$. Two days earlicr a brief observation showed it $10 \mathrm{lxc}$ at 0.5 mJy. The emission was unpolarized (below $5 \%$ ) and decayed slowly for alsout 6 hours. UV Cet has been observed far more frequently, but has never shou st such an ouburst.

\section{Olher quiescent sources}

UV Ceti is the best observed guiescent emilter. T"iere are unforturatcly no oller candidates yet identified which are such reliable and strong quiescent sources. We have compiled a list of some $83 \mathrm{M}$ dwarves observed at the VLA, of which 27 have unlambiguous detections (here both stars in binaries wider than 1" are couned separalcly; White, Kundu and Jackson, 1988, in preparation). The ability to decide whether a star is a quicscent source or not depends 
critically on having both long observations and sufficient flux in order to study the time variability of the source and identify the contribution of flaring to the flux. Most of the detected stars have not had such long observations, and consequently we are unable to reliably identify more than a few good quiescent candidates. Many of the stars could have quiescent emission at a level slightly below that of UV Cet and not be recognized as quicscent sources because they are more distant. Reliable sources of quiescent emission strong enough for detailed study are (IV Cel, ISQ Peg A, BYY Dra, and possibly AU Mic and the northem component of AT Mic. We expect that many more stars will be confirmed as quicscent sources when more observations have becen carrical out.

\section{Kallge of brightness temperatures}

lixact brightness temperatures for quiescent emission cannot be given since the sources are not resolved and we have no good idea of the source size. If we assume a source the size of the star, then UV Celi is reliably at a brightness temperature of several times $10^{8} \mathrm{~K}$ at $6 \mathrm{~cm}$. The strongest $6 \mathrm{~cm}$ quiescent emission yet seen was from EQ Peg A, which reached a brightness temperature of $210^{9}\left(\mathrm{R} / \mathrm{R}_{*}\right)^{-2} \mathrm{~K}$. Brightness temperatures of quiescent emission at $20 \mathrm{~cm}$ are often around this value, and values up to $10^{10} \mathrm{~K}$ have been claimed, although in that case the cmission was variable and may not have been the usual form of quicscent emission addressed here (Bastian and Bookbinder, 1987). At any rate, it is clear that brightness temperatures are often in the range where gyrosynchrotron emission is the likely emission mechanism. In view of the higher brightness temperatures at $20 \mathrm{~cm}$ than at $6 \mathrm{~cm}$, a non-1hermal electron conergy spectnum is implicd.

\section{Aclive region emission}

'The obvious interpretation of the outburst on EQ Peg is that the large flare injected energetic particles into loops above an active region, and these were responsible for the elevated levels of puiescent emission. The only problem with this is that after 6 hours of decay the radio emission suddenly jumped again without any sign of $\mathrm{X}$-ray emission. In any case, we assume that the quiescent radio emission comes from non-themal electrons trapped in loops alove active regions on the surface.

We argue that the true size of the radio-emilting region is of the order of the active region sia, which is smaller than a stellar radius. The argument is based on two observational results: the presence of strong magnetic ficlds covering a large fraction of the surface of these stiars (Sialr and l.insky, 1988), logether with the absence of any net longitudinal magnetic field component above about a few per cent of the average surface field in observations of the Zeeman effect (c.g., sec the review by Siar, 1987). This suggests that the magnetic field is present in a number of active regions smaller than a stellar radius, each with cancelling positive and negillive flux regions. If the magnetic field were dominated by a single large region, then at least at some phase of stellar rotation we would expect to see signs of polarization due to the lecman effect.

Since the active regions should be smaller than a stellar radius, it secms unlikely that the loops extend to any great height above the surface. On this basis, we do not expect that the radio source is several times larger than the stellar surface area, as has often appeared an attractive assumption since it reduces the brightness temperatures.

\section{Analytic models for dipolar region emission}

Can a relatively small active region account for the observed flux levels? To check this, we have developed simple analytic models for the flux from a buried dipole (White el al., 1988). We use the Dulk and Marsh (1982; Dulk, 1985) formulac for non-thermal gyrosynchrotron emission in the irequency range of 10 - 100 times the gyrofrequency. In the optically thich limit, we disume that the source can be approximated by a uniform brighuness temperature and a cerain linear dimension. The lalter is taken to be the height above the stellar surface at which the emission becomes optically thick, and the brightness temperature is taken to be the brightness temperature in that layer. We then assume that the source area is proportional to the spuare of the linear dimension, and use numerical integration of the Dulk and Marsh 
formulac to find the appropriate constant of proportionality. Since this model is only expected to give answers correct to within about a factor of 2 , we find that $2 \pi$ gives adequate results.

One way in which the model is an improvement on previous analylic work is that we allow for non-uniform source regions, i.c., we assume that both the density and the mangetic licld have power-law distributions with height above the surface, $B=B_{0}\left(r / r_{0}\right)^{-n}$ and $N=N_{1}$ $\left(\mathrm{r} / \mathrm{r}_{0}\right)^{-\mathrm{ml}}$. The lluxes and spectra depend on the power-law indices, and show a greater range of beliaviour than the homogeneous non-Uhennal gyrosynclinotron models. We have treated the cases of monopolar $(n=2)$ and dipolar $(n=3)$ magnetic fields, and unifom densily $(m=0)$ and hux-conserving $(m=n)$ density models. In Table I we show the frepuency spectral indices in the optically thick region of the spectrum of a dipolar active region viewed from atoove, for several parameters: the power-law index of the electmon energy distribution, $\delta$; the density index $\mathrm{m}$; and the magnetic field index $\mathrm{n}$.

The interesting feature of this table is that the predicted optically thick spectra are all much flatter than would be expected from the homogeneous fommulac, which predict spectral indices in a narrow range near 2.7 - 2.9. Indeed, for very hard energy spectra and a slow falloff of magnelic lield and particle density with height, r. pillive spectral indices can be oblained in the optically thick part of the spectrum.

Similarly simple analytic models can also be developed for the whically thin limit (hight frequencies), but these have the same spectral index as the homogeneous fomulic (1.2-0.98).

We have carried out numerical calculations of the flux from a dipoliar region using the Dulk and Marsh formulac with the exact geometry of a buried dipole, both to clieck the analylic formulac and to see whether a single active region of small size can indeed provide enough flux to explain the observations. We find that, for a range of plausible values of the critical parameters $\delta, n, m$, the surface density $N_{0}$, the surface magnetic field $B_{0}$, and the scalc size $r_{0}$ of the active region, the flux depends very strongly on the value of $\delta$, but that the observed fluxes caun be easily explained. That is, with true brightness temperalures in excess of $10^{9} \mathrm{~K}$, one only needs a source size much sillaller than the stellar surface area to explatin the observed tluxes, and the observed frequency spectra can easily be reproduced (of course, we have a number of free parameters in the models which can be varied). In facl, the predicted flux can be much larger than observed when $\delta=2$ (fluxes of around $100 \mathrm{ml}$ from UV Ceti, from an active region of dimension $10^{10} \mathrm{~cm}$ in size), duc to the fact that the source becomes optically thick far from the surface in this casc, producing both a large source and a large true brightness temperature. In general, the numerical calculations also show that the analylic formulac are accuratc to within a factor of beltcr than two, which we regard as surprisingly good.

However one result of these calculations is that for many plausible sicts of paramelers, particularly when the magnetic field strength is large, the optically thick layess are likely to be in regions where the harmonic numbers are 5-10. The Dulk and Marsh formulace on which Figures 1 and 2 are based are not valid in that frequency range. This affects many of the points, both optically thick and thin, at 5 and $15 \mathrm{Gll} z$ for $\delta \geq 3$.

Thus relatively small active regions can provide the observed puiesecn fluxes from $M$ Jwarf stars, and by varying the dependence of density and magnelic field with height above the active region we can also explain variable ratios of the $20 \mathrm{~cm}$ to $6 \mathrm{~cm}$ flux.

\section{From whence the electrons?}

In many ways, quiescent $M$ dware radio emission has more in common with solar radio flares than with solar quiescent cmission: both seen, to be due to non-thermal electrons radiating gyrosynchrotron emission in loop; above acive regions. However, in the solar case the duration of non-thermal radio emission after one injection of electrons is usually less than 30 minutes. In our interpretation of the EQ Peg event above, we suggested that a single episode of clectron injection occurred at the time of the X-ray flare, and this episode would then have produced stellar quiescent cmission lasting for many hours.

However, we have argued elsewhere based on the analogy with the Earh's radiation belts (Kundu et al., 1987) that one cannot ckpect electrons to remain in a stellar loop for longer than an hour before they precipitate into the denser lower regions of a stellar corona, and this is 
consistent with the shorter duration of solar non-thermal events. Thus long-lasting stellar events imply instead continual replenishment of the electrons. If indeed there are many active regions crowding the surface whose magnetic loops jostle one another in the corona, then continual reconnection events in the spirit of Parker's (c.g., 1988) ideas can casily be envisaged as providing a steady supply of non-thermal electrons. The $20 \mathrm{~cm}$ observations of EQ Peg during the event discussed above support this, in that they show flaring activily continuing throughout the whole day (although no flaring was evident at $6 \mathrm{~cm}$ ).

Another seldom-discussed question is how the radio-emilting particles coexist with the $X$-ray-cmilting corona. The X-ray-emilling material is at relatively high density, and if the non-thermial radio particles were to come into contact with it they would rapidly lose their energy. This stuggests that the non-thermal radio-emitting particles are on different loop syslems, and that their generation mechanism is also different from that which produces the X-ray corona. We are lead to a picture in which flaring occurs essentially independently at different levels of the corona, in agreement with observations that there is little correlation between radio, X-ray or optical flares.

UV Cet has been observed more frequently than EQ Peg, and has shown a three-fold variability in its $6 \mathrm{~cm}$ quiescent llux, but no outbursts such as the twenty-fold increase by EQ Peg $A$. llow do we reconcile this? Since it is possible to explain EQ Peg's emission with a single active region, it simply implies that UV Cet's active regions are more uniform in their presence on the visible disc, and more regular in their radio behaviour. Equivalently, we suggest that UV Cel's emission is due to a number of active regions, whereas the large outburst on IEQ Peg was due to a single active region.

A prediction following from this is that al optically thin frequencies UV Cel's quiescent emission is less likely to show polarization than an outburst event such as EQ Peg A's, since the former may come from summing over several regions whereas the latter comes from a single active region and ariy geometric asymmetry in the source structure may well show up as polarization, particularly al optically thin frequencies.

\section{References}

13:Lstian, T.S., and J.A. Bookbinder, 1987, Nature, 326, 678.

Dulk, G.A., 1985, Ann. Rev. Astr. Ap. 23, 169.

Dulk, G.A., and K.A. Marsh, 1982, Ap. J. 259, 350.

Gary, D.E., and J.L. Linsky, 1981, Ap. J. 250, 284.

Kundu, M.R., P.D. Jackison, S.M. White and M. Mclozzi, 1987. Ap. J. 312, 822.

Kundu, M.R., R. Pallivicini, S.M. White and P.D. Jackson, 1988, Astr. Ap. 195, 159

Parker, E.N., 1988, Ap. J. 330, 474.

Salar, S., J.L. Linsky and M.S. Giampapa, 1987, Observational Astrophysics with High Precision Dara, Licge, Belgium.

Saar, S., 1987, Prociedings of the Fifth Cambridge Workshop on Cool Stars, Stellar Systems and the Sun, Springer-Verlag (Berlin), p. 10.

White, S.M., M.R. Kundu and P.D. Jackson, 1988, submitted to Astr. Ap.

\section{Table 1}

Optically thick spectral indices for a range of electron energy

distributions, magnetic field variation and density variation.

\begin{tabular}{|c|c|c|c|c|}
\hline$v$ & $\delta=2$ & $\delta=3$ & $\delta=4$ & $\delta=5$ \\
\hline$n=2, m=0$ & -0.42 & 0.04 & 0.26 & 0.39 \\
$n=2, m=2$ & 0.70 & 0.77 & 0.81 & 0.83 \\
$n=3, m=0$ & 0.41 & 0.68 & 0.82 & 0.90 \\
$n=3, m=3$ & 1.18 & 1.21 & 1.23 & 1.24 \\
\hline
\end{tabular}

